\title{
The Place of DPP-4 Inhibitors in the Treatment Algorithm of Diabetes Type 2: a Systematic Review of Cost-effectiveness Studies
}

Baptista A, Teixeira I, Romano S, Carneiro AV, Perelman J.

Published in: Eur J Health Econ. 2017;18:937-65.

\section{Objective}

To conduct a systematic review of cost-effectiveness, cost-utility, and cost-benefit studies of DPP-4 inhibitors for diabetes treatment versus other antidiabetics.

\section{Methods}

Three investigators searched the CRD York, Tufts CEA Registry, and MEDLINE databases through 2015. We reviewed all potentially relevant titles and abstracts, and screened full-text articles, according to inclusion criteria. We established a quality score for each study based on a 35-item list.

\section{Results}

A total of 295 studies were identified, of which 20 were included. The average quality score was 0.720 on a $0-1$ scale. All studies were performed in highand middle-income countries, using a 3rd-party payer perspective and randomized clinical trials to measure effectiveness. Sitagliptin, saxagliptin and vildagliptin had an ICER below 25,000 €/QALY, as second-line and as add-ons to metformin, in comparison to sulfonylureas. When compared with sitagliptin, liraglutide (GLP-1 receptor agonist) had an ICER of up to 22,724 €/QALY for the 1.2-mg dosage, and up to $32,869 € / \mathrm{QALY}$ for the 1.8-mg dosage. Insulin glargine was dominant when compared with sitagliptin.

\section{Conclusions}

According to the WHO threshold applied to the country and year of each study, DPP-4 inhibitors were highly cost-effective as second-line, as addons to metformin, in comparison with sulfonylureas. More recent therapies (GLP-1 receptor agonists and insulin glargine) were highly cost-effective in comparison to DPP-4 inhibitors. These results were ob- tained, however, on the basis of a limited number of studies, relying on the same few clinical trials, and financed by manufacturers. Further independent research is needed to confirm these findings. 